OPEN ACCESS

Edited by:

Masakazu Toi,

Kyoto University,

Japan

Reviewed by:

Takayuki Ueno,

Kyorin University, Japan

Minoru Miyashita,

Tohoku University, Japan

*Correspondence: Sherene Loi

sherene.loi@petermac.org

Specialty section:

This article was submitted

to Women's Cancer,

a section of the journal

Frontiers in Oncology

Received: 31 May 2017

Accepted: 04 July 2017

Published: 03 August 2017

Citation:

Wein L, Savas P, Luen SJ, Virassamy $B$, Salgado $R$ and Loi $S$ (2017) Clinical Validity and Utility of

Tumor-Infiltrating Lymphocytes in

Routine Clinical Practice for Breast

Cancer Patients: Current and Future

Directions.

Front. Oncol. 7:156.

doi: 10.3389/fonc.2017.00156

\section{Clinical Validity and Utility of Tumor-Infiltrating Lymphocytes in Routine Clinical Practice for Breast Cancer Patients: Current and Future Directions}

\author{
Lironne Wein ${ }^{1}$, Peter Savas', Stephen J. Luen ${ }^{1}$, Balaji Virassamy ${ }^{1}$, Roberto Salgado ${ }^{1,2}$ \\ and Sherene Loi ${ }^{1,3 *}$ \\ ${ }^{1}$ Peter MacCallum Cancer Centre, University of Melbourne, Melbourne, VIC, Australia, ${ }^{2}$ Department of Pathology, GZA \\ Ziekenhuizen, Antwerp, Belgium, ${ }^{3}$ University of Melbourne, Melbourne, VIC, Australia
}

The interest in tumor-infiltrating lymphocytes (TILs) as a prognostic biomarker in breast cancer has grown in recent years. Biomarkers must undergo comprehensive evaluation in terms of analytical validity, clinical validity and clinical utility before they can be accepted as part of clinical practice. The International Immuno-Oncology Biomarker Working Group has developed a practice guideline on scoring TILs in breast cancer in order to standardize TIL assessment. The prognostic value of TILs as a biomarker in early-stage breast cancer has been established by assessing tumor samples in thousands of patients from large prospective clinical trials of adjuvant therapy. There is a strong linear relationship between increase in TILs and improved disease-free survival for triple-negative and HER2-positive disease. Higher levels of TILs have also been associated with increased rates of pathological complete response to neoadjuvant therapy. TILs have potential clinical utility in breast cancer in a number of areas. These include prediction of responders to immune checkpoint blockade, identification of primary HER2-positive and triple-negative patients who have excellent prognoses and may thus be appropriate for treatment de-escalation, and potentially incorporation into a neoadjuvant endpoint which may be a better surrogate maker for drug development.

Keywords: tumor-infiltrating lymphocytes, breast cancer, cancer biomarkers, antitumor immunity, checkpoint inhibitors

\section{INTRODUCTION}

It has been well established that the immune system plays a crucial role in host defense against tumor progression. Unlike solid tumor types such as melanoma, breast cancer is not usually considered an immunogenic; however, recent evidence suggests that tumor immunogenicity is important in the biology of breast cancer and its response to treatment. Tumor-infiltrating lymphocytes (TILs) have provided insight into the immunogenicity of breast tumors and have been shown to be useful as a prognostic biomarker in early-stage triple-negative and HER2-positive breast cancer. 


\section{BACKGROUND: THE IMMUNE SYSTEM AND TILS IN BREAST CANCER}

Cancer immunoediting is believed to be a central component in the evolutionary processes that shape an established tumor, and therefore also plays a critical role in the determination of the resulting immunogenic phenotype $(1,2)$. Immunoediting comprises three processes, namely, elimination, equilibrium, and escape. Elimination encompasses the concept of immunosurveillance, where the host immune system recognizes tumor antigens and destroys the developing tumor. It is characterized by the presence of $\mathrm{CD} 8+\mathrm{T}$ lymphocytes, $\mathrm{CD} 4+$ type 1 helper $\mathrm{T}$ lymphocytes, and natural killer cells. When this elimination process is not successful, the tumor enters the equilibrium phase, whereby the tumor cell clones with superior survival abilities are selected in a Darwinian fashion. Escape occurs when the immunologically sculpted tumor proliferates in an uncontrolled manner and becomes clinically detectable. During escape, FOXP3+ $\mathrm{T}$ regulatory lymphocytes, CD4+ type $2 \mathrm{~T}$ helper lymphocytes, and myeloid-derived suppressor cells can be seen in the tumor microenvironment (3).

Tumor-infiltrating lymphocytes are immune cells that have been observed in many solid tumors, including breast cancer. They are a population of cells comprising a mixture of cytotoxic $\mathrm{T}$ cells and helper $\mathrm{T}$ cells, as well as B cells, macrophages, natural killer cells, and dendritic cells. Whilst not discussed extensively in this manuscript, gene expression and flow cytometry profiles demonstrate that TIL quantity, as assessed semiquantitatively on a hematoxylin and eosin (H\&E) stained slide, represents a surrogate for a pre-existing favourable host anti-tumor activated $\mathrm{T}$ cell response $(4,5)$.

Tumor-infiltrating lymphocytes are more commonly observed at higher levels in triple-negative and HER2-positive tumors compared with estrogen receptor positive, HER2-negative breast cancer (6-8). This indicates that TNBC and HER2-positive subtypes are more immunogenic, and for reasons yet to be fully elucidated are able to generate more robust $\mathrm{T}$ cell anticancer responses. Lately the clinical importance of the presence of TILs has been reported, with associations with improved prognosis and higher response rates to neoadjuvant therapy in the earlystage setting $(9,10)$.

\section{WHAT MAKES A GOOD BIOMARKER?}

Biomarkers in cancer must undergo thorough evaluation in terms of analytical validity, clinical validity, and clinical utility before they can be adopted as part of clinical practice (11). Analytical validity refers to the robustness of the test, in terms of accuracy, reliability, and interobserver reproducibility (12). The clinical validity relates to the extent to which the biomarker is associated with prognosis or response to treatment and refers to the ability of a tumor biomarker to divide one population into groups that differ either biologically or clinically. Importantly, clinical validity alone is insufficient to recommend that the biomarker be used to guide clinical treatment decisions. Clinical utility is the usefulness of the biomarker in terms of directing clinical decisions and patient management.

\section{ANALYTICAL VALIDITY}

As the interest in TILs as an immunological biomarker grows, so too does the need for standardizing TIL evaluation. The International Immuno-Oncology Biomarker Working Group has developed a practice guideline and tutorial on how to score TILs in breast cancer (13). Currently, most clinical research groups world wide interested in TIL evaluation in breast cancer are using this guideline in their studies, which will promote comparability of studies. This will avoid a plethora of different methods assessing the same biomarker, as has been the experience with Ki-67, although for Ki-67 international efforts at standardization are also underway (14).

Tumor-infiltrating lymphocytes in breast cancer lesions can be seen in both the intratumoral and stromal areas. Intratumoral TILs have cell-to-cell contact with carcinoma cells, while stromal TILs are located in the fibrous stroma adjacent to the tumor cells. Evaluation of intratumoral TILs is generally more difficult and less reproducible than evaluation of stromal TILs but the separation of these two variables may have biological connotations. The current recommendation is therefore to use the percentage of stromal TILs as the principal parameter, where the numerator is the area occupied by TILs and the denominator is the total stromal area, not the number of stromal cells. All mononuclear cells (lymphocytes and plasma cells) are scored, but polymorphonuclear white blood cells are excluded (13).

The guidelines of the International Immuno-Oncology Working Group clearly state that TILs should be assessed as a continuous variable rather than as a categorical variable (13). Having TILs documented as a continuous variable enables it to be implemented in algorithms encompassing other prognostic clinical-pathological variables. Loi and colleagues have demonstrated that TILs incorporated as such in an algorithm containing nodal status, tumor size, and age enables making predictions of the 5-year predicted distant disease-free survival (15) (Figure 1). Where a binary variable is needed, the term lymphocyte predominant breast cancer (LPBC) can be used to describe cancers which comprise more lymphocytes than tumor cells. Previous reports of LPBC have variably utilized a threshold between 50 and $60 \%$ stromal lymphocytes; however data since the original publications suggest that this may not be the best cutpoint as this level of immune infiltration represents less than $10 \%$ of primary breast cancers. This is considerably lower than that of melanoma and non-small cell lung cancer in which this level of lymphocytic infiltration is present in around $25 \%$ of tumors.

Tumor-infiltrating lymphocytes can be easily and are most commonly assessed using light microscopy of H\&E stained slides. More complex techniques have been used, such as flow cytometry (16) and multicolor immunohistochemical staining with multispectral imaging (17), as well as gene expression analysis measured using quantitative polymerase chain reactions, gene expression microarrays, and RNA sequencing $(18,19)$. These 


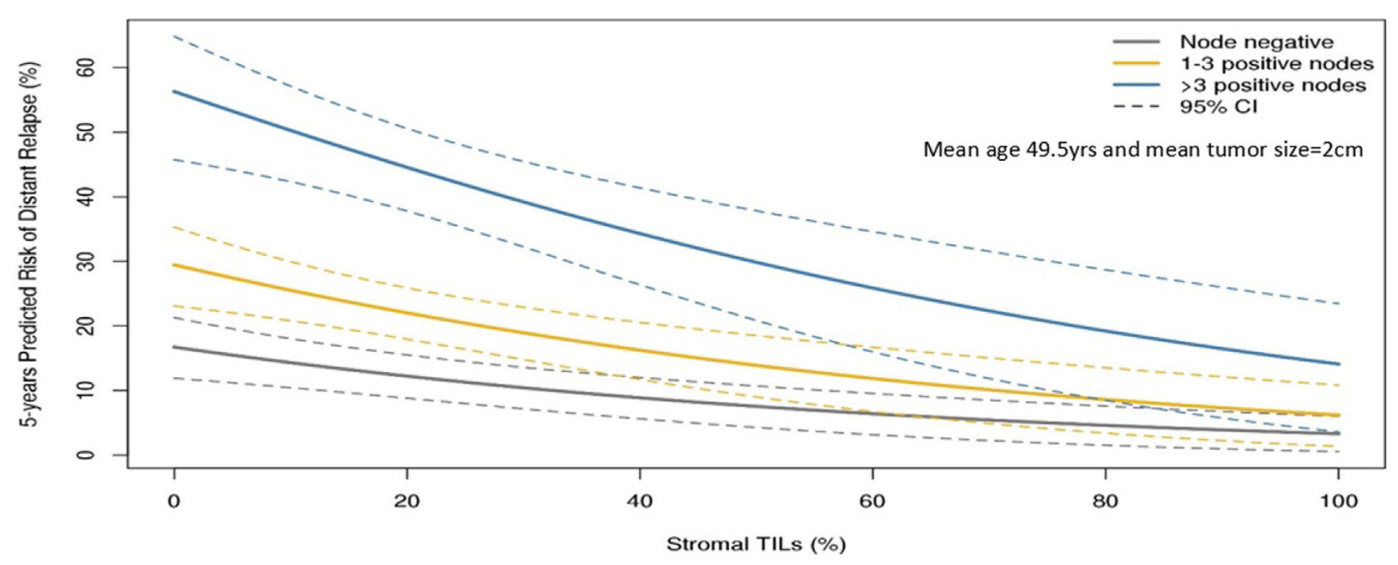

FIGURE 1 | Model to predict risk of distant recurrence at 5 years (\%) by tumor-infiltrating lymphocytes (TILs) and nodal status by tumor size and age. The predicted rate of distant recurrence on the $y$ axis as a continuous function of the level of stromal TILs in the primary tumor on the $x$ axis is presented according to nodal status after adjuvant chemotherapy. This prognostic algorithm is for TNBC and assumes a mean age of 49.5 years and mean tumor size of $2 \mathrm{~cm}$. Loi et al., San Antonio Breast Cancer Symposium 2015 (15).

techniques can provide more information regarding the different lymphocyte subpopulations; however, gene expression data are limited with regard to identifying specific T cell subsets. All of these techniques remain in the research setting due to cost, lack of robust analytical validity, and requirements for certain types of tissue processing.

The International Immuno-Oncology Working Group conducted two ring studies to evaluate interobserver variability of TIL assessment (20). The aim was to calculate the interclass correlation coefficient (ICC) for assessment of TILs by different pathologists. In the first ring study, 34 pathologists assessed 60 slides for stromal TILs. While the interobserver agreement was relatively good, the prespecified endpoint of an ICC above 0.7 was not reached (ICC: 0.70; 95\% CI: 0.62-0.78). A software-guided image evaluation system was developed for the second ring study, where 28 pathologists evaluated 60 slides. The ICC improved to 0.89 (95\% CI: 0.85-0.92). This suggests that TIL evaluation can be reproducible among pathologists and that computer-assisted systems may be valuable in this regard. A study by Swisher et al. (21) also found that TIL evaluation can be undertaken with acceptable interobserver agreement. Four pathologists assessed 75 TNBC specimens. The kappa statistic for interobserver agreement was 0.57 , which was interpreted as moderate agreement.

Nevertheless, some hurdles still remain with regard to standardizing TIL assessment. For example, TIL infiltration patterns within a single tumor can be quite heterogeneous (Figure 2). It is well known that the eye of the pathologist may not be able to quantify reliably the spatial variation in infiltration patterns. In this respect, machine learning algorithms may potentially be of added value (22). Regardless, TIL evaluation by H\&E remains the most pragmatic for many community laboratories in the absence of data supporting other methods.

As the majority of experience in TIL assessment in breast cancer has been in primary lesions, less is known about immune influences in the advanced metastatic setting. Important differences in the immune infiltrates between primary tumors and

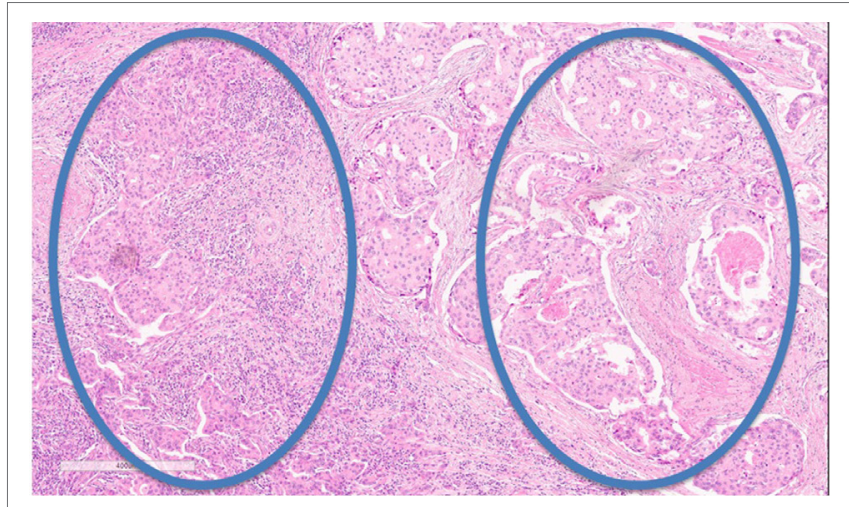

FIGURE 2 | Tumor-infiltrating lymphocyte (TIL) heterogeneity. Zone with high TILs on the left, zone with low TILs on the right. Magnification 100x.

metastases have recently been reported. Studies of paired primary and metastatic lesions have noted significantly lower levels of TILs in metastases (23-25) (Figure 3). This is in keeping with lower TIL levels described in metastases in lung cancer (26) and renal cell carcinoma (27). The TILs working group guideline (13) methodology for TIL assessment has been used to evaluate TILs in the advanced setting in a retrospective analysis of the CLEOPATRA study (28). This study also found lower TIL levels in metastatic lesions than in their paired primaries, although there were not enough paired samples to adequately power this comparison. The site of metastases may also have an important influence on the immune infiltrate, with higher TIL levels having been observed in lung metastases than in bone, liver, or skin lesions. The International Immuno-Oncology Working Group will soon publish guidelines on scoring TILs in the metastatic setting and in the residual disease setting. Overall, these observations lend evidence to the hypothesis that breast cancer must adapt and learn how to evade the immune system as well as cause significant immunosuppression in the host in order to progress 


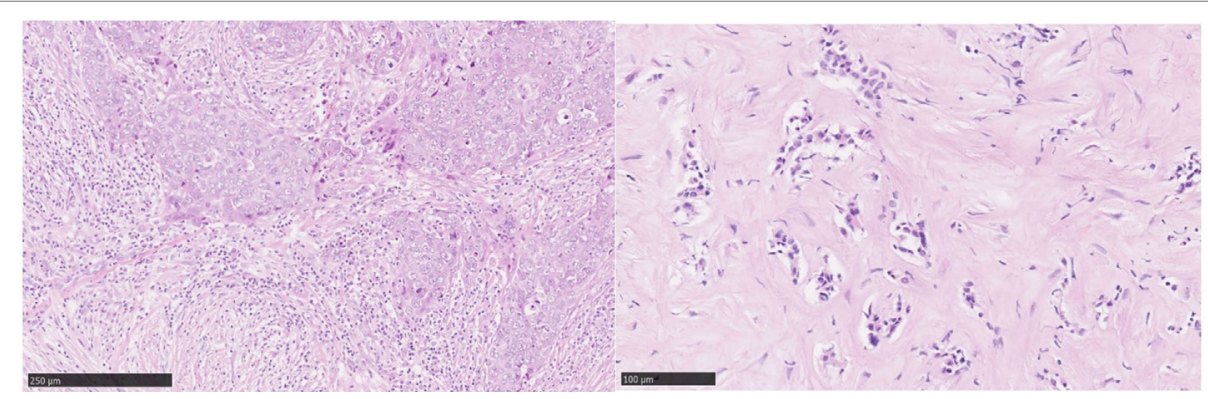

FIGURE 3 | Tumor-infiltrating lymphocytes (TILs): primary versus metastatic disease. Case study: breast primary with high number of TILs (left), pleural metastasis with no TILs (right). Magnification 100x (left), 200x (right).

to the advanced disease setting. This has implications for the development of immunotherapies in advanced breast cancer.

\section{CLINICAL VALIDITY}

\section{The Prognostic Value of TILs in Early- Stage Breast Cancer}

The prognostic value of the TILs biomarker in early-stage breast cancer has been established by assessing tumor samples in patients from large clinical trials (Table 1). There has been shown to be a strong linear relationship between increase in TILs and improved recurrence-free survival for triple-negative breast cancer and HER2-positive breast cancer (10). In the first TIL analysis of node-positive primary breast cancers from the BIG 02-98 trial (7), over 2,000 breast cancer samples were examined for TILs. In triple-negative breast cancer, each $10 \%$ increase in intratumoral and stromal TILs was associated with a 17 and $15 \%$ reduced risk of relapse, respectively, and 27 and $17 \%$ reduced risk of death, respectively, regardless of chemotherapy type.

These findings were validated in an analysis of 935 early-stage breast cancer samples from the FinHER trial (29). In triplenegative breast cancer and in HER2-positive patients randomized to trastuzumab, each $10 \%$ increase in TILs was associated with decreased distant recurrence. In the HER2-positive group, higher TILs were also associated with greater magnitude of benefit from the addition of adjuvant trastuzumab. A study looking at gene expression profile of TILs and benefit of trastuzumab in the NSABP B-31 HER2-positive breast cancer clinical trial also found that patients with high expression of TILs genes derived significantly more benefit more from trastuzumab than patients with low-TIL expression (30).

In a study of 506 triple-negative tumors from the adjuvant ECOG trials E2197 and E1199, stromal TILs, as a continuous variable, were associated with improved prognosis (31). Similarly, in a recent analysis of 647 TNBC samples, TILs were associated with an improved prognosis at a median follow-up of 6.9 years (32). For every 10\% increase in TILs, the breast cancer-free interval risk reduction was $13 \%$ (HR 0.87, 95\% CI: $0.79-0.95, P=0.003)$. Higher TIL levels were also associated with better disease-free survival and overall survival, with risk reductions of $11 \%(P=0.005)$ and $17 \%(P<0.001)$, respectively.
Loi et al. conducted a pooled analysis with individual patient data from 991 TNBC patients from 6 randomized clinical trials (33). Each $10 \%$ increase in stromal TILs was associated with a $14 \%$ relative reduction in invasive disease-free survival $(\mathrm{HR}=0.86$, 95\% CI: $0.80-0.93, P<0.0001)$ Figure 1 and a $17 \%$ relative reduction in deaths $(\mathrm{HR}=0.83,95 \% \mathrm{CI}: 0.76-0.91, P=0.0001)$. TILs therefore represent the first biological prognostic factor for primary TNBC that has been shown to be reproducible and also add robust significant addictive prognostic value to current clinicopathological factors. Consideration into inclusion into staging systems such as AJCC (34) should be considered in the future.

Ali et al. (8) analyzed samples from four studies including more than 12,000 patients. They found that in ER-negative tumors (ER-negative/HER2-positive and triple-negative), the presence of stromal and intratumoral CD8+ lymphocytes was associated with improved breast cancer specific survival. However, in ER-positive breast cancer, CD8+ lymphocytes were not found to be associated with breast cancer-specific survival.

The studies discussed above provide robust and consistent evidence that TILs can be used as a prognostic marker in earlystage triple-negative breast cancer and in some patients with HER2-positive disease. At present, the significance of TILs in ER-positive HER2-negative tumors has not been established.

\section{TILs and Response to Neoadjuvant Chemotherapy}

Tumor-infiltrating lymphocytes have been associated with higher rates of pathological complete response (pCR) to neoadjuvant therapy (Table 2). Denkert et al. analyzed 1,058 pretreatment biopsies from two neoadjuvant anthracycline and taxane-based trials and found that the level of intratumoral lymphoctytes was significantly associated with pCR rates (35). In the GeparDuo cohort, the overall pCR rate was $12.8 \%$, while in patients with increased intratumoral lymphocytes $(>10 \%)$ it was $31 \%$. In the subgroup of LPBC, defined as $>60 \%$ of either stromal or intratumoral lymphocytes, pCR rate was $41.7 \%$. Similarly, in the GeparTrio cohort, the odds ratio for pCR increased with the extent of TILs. Tumors without any TILS had a PCR rate of $7.2 \%$, while those with LPBC had a pCR rate of $40 \%$.

Tumor-infiltrating lymphocytes have been associated with achieving response to neoadjuvant HER2-targeted therapies in several studies. In an analysis of the GeparQuattro trial, each 
TABLE 1 | Adjuvant trials that have assessed tumor-infiltrating lymphocytes (TILS).

\begin{tabular}{|c|c|c|c|c|}
\hline Trial & Treatments & Subtype & $n$ & Recurrence end points \\
\hline BIG 2-98 (7) & $\begin{array}{l}\text { Doxorubicin } \\
\text { Cyclophosphamide } \\
\text { CMF } \\
\text { Docetaxel }\end{array}$ & $\begin{array}{l}\mathrm{ER}+/ \mathrm{HER} 2- \\
\mathrm{HER} 2+ \\
\mathrm{TNBC}\end{array}$ & $\begin{array}{r}1,079 \\
297 \\
256\end{array}$ & $\begin{array}{l}\text { Not significant } \\
\text { Not significant } \\
\text { For each } 10 \% \text { increment of sTILs: DFS, HR =0.84 (95\% Cl: } 0.74-0.98, P=0.025)\end{array}$ \\
\hline FinHER (29) & $\begin{array}{l}\text { Docetaxel } \\
\text { Vinorelbine } \\
\text { FEC } \\
\pm \text { Trastuzumab }\end{array}$ & $\begin{array}{l}\mathrm{ER}+/ \mathrm{HER} 2- \\
\mathrm{HER} 2+ \\
\mathrm{TNBC}\end{array}$ & $\begin{array}{l}591 \\
209 \\
134\end{array}$ & $\begin{array}{l}\text { Not significant } \\
\text { Predictive for higher trastuzumab benefit }(P \text { interaction }=0.025) \\
\text { For each } 10 \% \text { increment of sTILs: DDFS, HR=0.79 (95\% Cl: } 0.64-0.98, P=0.032)\end{array}$ \\
\hline E2197 and E1199 (31) & $\begin{array}{l}\text { Doxorubicin } \\
\text { Cyclophosphamide } \\
\text { Docetaxel }\end{array}$ & TNBC & 481 & For each $10 \%$ increment of sTILs: DFS, HR = $0.84(95 \% \mathrm{Cl} 0.74-0.95, P=0.005)$ \\
\hline IBCSG 22-00 (32) & $\begin{array}{l}\text { CMF } \\
\text { Anthracycline } \\
\text { Taxanes } \\
\text { CM-maintenance }\end{array}$ & TNBC & 647 & $\begin{array}{l}\text { For each } 10 \% \text { increment of sTILs: Breast cancer-free interval, } \mathrm{HR}=0.87 \\
(95 \% \mathrm{Cl} 0.79-0.95, P=0.003)\end{array}$ \\
\hline Pooled analysis (33) & $\begin{array}{l}\text { Anthracycline } \\
\text { Taxane }\end{array}$ & TNBC & 991 & $\begin{array}{l}\text { Studies included ECOG2197, ECOG1199, BIG2-98, FinHER, Gustave Roussy } \\
\text { For each 10\% increment of sTILs: IDFS HR }=0.86 \text { (95\% Cl: } 0.80-0.93, P<0.0001)\end{array}$ \\
\hline Total patients & & & 4,685 & \\
\hline
\end{tabular}

DDFS, distant disease-free survival; IDFS, invasive disease-free survival; DFS, disease-free survival; iT-CD8, intratumoral CD8; S-CD8, stromal CD8; sTIL, stromal TIL.

TABLE 2 | Neoadjuvant trials that have assessed tumor-infiltrating lymphocytes (TILs).

\begin{tabular}{|c|c|c|c|c|}
\hline Trial & Treatments & Subtype & $n$ & pCR \\
\hline GeparDuo (35) & $\begin{array}{l}\text { Doxorubicin } \\
\text { Docetaxel } \\
\text { Cyclophosphamide }\end{array}$ & All & 218 & OR 1.38 of pCR per $10 \%$ iTILs (95\% Cl: $1.08-1.78, P=0.012)$ \\
\hline GeparTrio (35) & $\begin{array}{l}\text { Doxorubicin } \\
\text { Docetaxel } \\
\text { Cyclophosphamide } \\
\text { Vinorelbine } \\
\text { Capecitabine }\end{array}$ & All & 840 & OR 1.21 of pCR per $10 \%$ iTILs (95\% Cl: 1.08-1.35, $P=0.001)$ \\
\hline NeoALTTO (38) & $\begin{array}{l}\text { Trastuzumab } \\
\text { Lapatinib } \\
\text { Paclitaxel } \\
\text { FEC }\end{array}$ & HER2+ & 387 & $\begin{array}{l}\text { Every } 1 \% \text { increase in TILs was associated with a } 3 \% \text { decrease in the rate of an } \\
\text { event }[\mathrm{HR} 0.97(95 \% \mathrm{Cl}: 0.95-0.99 ; P=0.002)] \\
\text { pCR: TILs }>5 \% \text { associated with higher pCR rates [OR } 2.60(95 \% \mathrm{Cl} \text { : } \\
1.26-5.39 ; P=0.01)]\end{array}$ \\
\hline GeparQuattro (36) & $\begin{array}{l}\text { Epirubicin } \\
\text { Cyclophosphamide } \\
\text { Docetaxel } \\
\text { Capecitabine } \\
\text { Trastuzumab }\end{array}$ & $\mathrm{HER} 2+$ & 156 & OR 1.16 of pCR per $10 \%$ sTILs $(95 \% \mathrm{Cl}: 1.01-1.32, P=0.038)$ \\
\hline CHER-LOB (37) & $\begin{array}{l}\text { Trastuzumab and/or lapatinib } \\
\text { Paclitaxel } \\
\text { FEC }\end{array}$ & HER2+ & 105 & $\begin{array}{l}\text { Each } 10 \% \text { increase in } \mathrm{iTIL} \text { and STIL associated with a higher probability of a } \\
\text { pCR (adjusted OR: } 2.64,95 \% \mathrm{Cl}: 1.46-4.79, P=0.001 \text { and } 1.3295 \% \mathrm{Cl} \text { : } \\
\text { 1.08-1.6, } P=0.006 \text {, respectively) }\end{array}$ \\
\hline GeparSixto (19) & $\begin{array}{l}\text { Paclitaxel } \\
\text { Liposomal doxorubicin } \\
\text { Carboplatin } \\
\text { Bevacizumab } \\
\text { Trastuzumab }\end{array}$ & $\begin{array}{l}\text { HER2+ and } \\
\text { TNBC }\end{array}$ & 580 & $\begin{array}{l}\text { OR } 1.2 \text { of pCR per } 10 \% \text { sTILs ( } 95 \% \text { Cl: } 1.11-1.29, P<0.001) \\
\text { Significant test for interaction between increased TILs and response to } \\
\text { carboplatin therapy }\end{array}$ \\
\hline GeparQuinto (39) & $\begin{array}{l}\text { Epirubicin } \\
\text { Cyclophosphamide } \\
\text { Taxane } \\
\text { Everolimus }\end{array}$ & $\mathrm{ER}+$ and $\mathrm{TNBC}$ & 313 & OR 1.2 of pCR per $10 \%$ sTILs (95\% Cl: $1.0-1.3, P=0.01)$ \\
\hline $\begin{array}{l}\text { EORTC } 10994 \text { and } \\
\text { BIG 00-01 (40) }\end{array}$ & $\begin{array}{l}\text { FEC } \\
\text { Docetaxel }\end{array}$ & ER- & 111 & $\begin{array}{l}\text { High gTILs: pCR } 74.2 \% \\
\text { Low gTILs: pCR } 31.3 \% \\
\text { OR } 6.42 \text { of pCR for high versus low gTILs ( } 95 \% \text { Cl: } 2.08-19.83, P=0.001)\end{array}$ \\
\hline
\end{tabular}

pCR, pathological complete response; iTIL, intratumoral TIL; sTIL, stromal TIL; gTIL, gene expression surrogate TIL. 
$10 \%$ increment in TILs was found to be associated with higher responses to neoadjuvant trastuzumab and chemotherapy (36). Similarly, in a study of 105 HER2-positive tumors from the CHER-LOB trial (37), both intratumoral and stromal TILs were associated with a higher pCR rates (OR: 2.64, 95\% CI: 1.46-4.79, $P=0.001$ and $1.32,95 \% \mathrm{CI}: 1.08-1.6, P=0.006$, respectively).

Additionally, in the NeoALLTO study (38) of HER2-positive patients treated with neoadjuvant HER2-targeted therapies and chemotherapy, TIL levels greater than $5 \%$ were associated with higher rates of pCR. This study also found the presence of TILs at diagnosis to be a positive, independent prognostic marker. There was a linear relationship between TILs and event-free survival, with every $1 \%$ increase in TILs being associated with a $3 \%$ decrease in the rate of an event.

The GeparSixto trial investigated the effect of adding carboplatin to an anthracycline-plus-taxane combination in triplenegative and HER2-positive tumors. Evaluation of these tumors demonstrated that increased levels of stromal TILs and LPBC were associated with higher rates of pCR (19). pCR rates in LPBC tumors were significantly higher with the addition of carboplatin, with carboplatin increasing the odds of pCR 3.71-fold in LPBC tumors compared with a 1.01-fold increase in non-LPBCs.

Lymphocytic infiltrate has also been shown to be associated with response to chemotherapy in HER2-negative disease. In an analysis of 313 biopsies from a substudy of the neoadjuvant GeparQuinto trial, LBPC had a significantly higher pCR rate compared to non-LPBC (36.6 versus 14.3\%) (39). In an exploratory analysis, pCR rates were analyzed according to hormone receptor status. In hormone receptor positive tumors, the $\mathrm{pCR}$ rate for LPBC was $28.2 \%$, while it was only $8.2 \%$ for non-LPBC tumors. Use of TILs as a predictive marker in the setting of hormone receptor-positive HER2-negative disease requires further validation.

West et al. examined 111 ER-negative tumors from patients treated with neoadjuvant anthracycline-based regimens (40). Pathologic complete responses were observed in 74\% of TIL-high patients and $31 \%$ of TIL-low patients (odds ratio 6.33; 95\% CI: 2.49-16.08; $P<0.0001)$.

The association of different subtypes of TILs with achieving response to neoadjuvant chemotherapy has been investigated in
TNBC (41). It was found that a high CD8+/FOXP3+ ratio was a strong predictor of $\mathrm{pCR}$. This result is consistent with the immunoediting hypothesis, as this TIL composition is representative of the elimination process.

Note that while a binary LPBC value was used commonly in these studies, present recommendations currently do not propose a specified cutoff for neoadjuvant patients. These findings do suggest that patients with low TILs on their baseline core-biopsy may need to be observed very carefully during their neoadjuvant therapy or perhaps baseline TILs levels could facilitate a decision to go straight to surgery (where possible).

\section{TILs in Residual Disease}

Tumor-infiltrating lymphocytes in residual disease after neoadjuvant therapy have been shown to be associated with a more favorable prognosis (Table 3). In an analysis of 278 TNBC specimens post neoadjuvant treatment, Dieci et al. found the 5 -year overall survival for high-TIL patients was 91\%, compared to $55 \%$ for low-TIL patients (42). Similarly, in a cohort of 111 TNBC patients with residual disease following neoadjuvant chemotherapy, a strong positive linear association was observed between TILs in the residual disease specimen and both recurrence-free survival and overall survival (43).

\section{The Prognostic Value of TILs in Metastatic Breast Cancer}

Tumor immunogenicity is hypothesized to decrease in the advanced setting (Table 4). This is based on the theory of immunoediting (1), and observations that metastatic tumors have lower levels of TILs than their matched primary tumors $(24,44)$. The prognostic value of TILs in advanced HER2-positive breast cancer has recently been examined (28). Six hundred and seventy-eight tumor samples from the CLEOPATRA study were assessed for TILs. Fifty eight of these were from metastatic sites. Twenty patients had paired primary and metastatic samples. There was no significant association between TIL levels and progression-free survival; however, for overall survival, each $10 \%$ increase in TILs was significantly associated with prolonged overall survival. This finding expands the prognostic role of TILs

TABLE 3 | Tumor-infiltrating lymphocytes (TILs) in residual disease post neoadjuvant treatment.

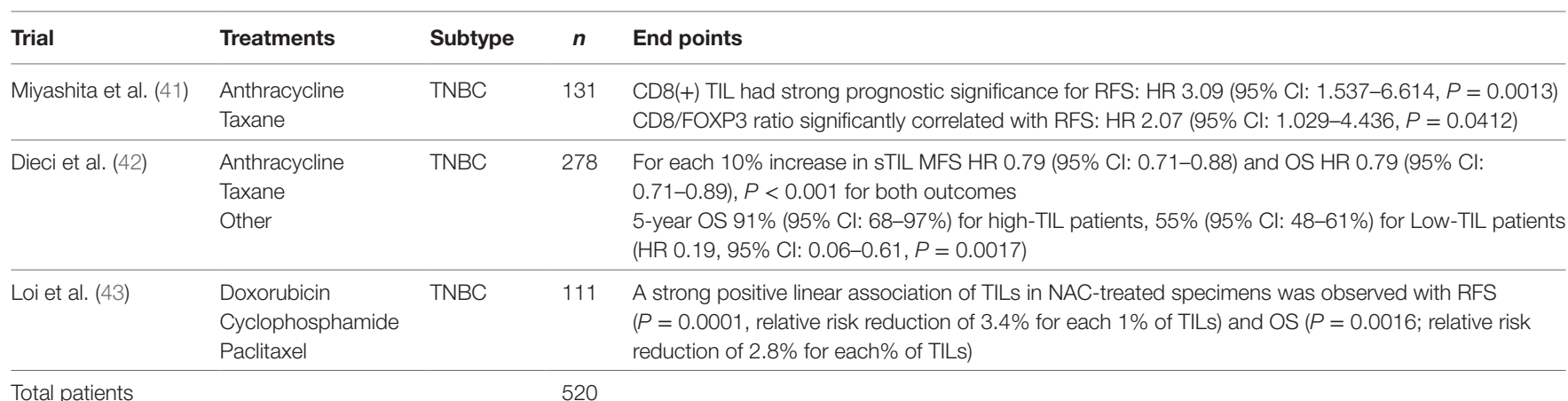


TABLE 4 | Tumor-infiltrating lymphocytes (TILs) in metastatic disease.

\begin{tabular}{|c|c|c|c|c|}
\hline Trial & Treatments & Subtype & $n$ & Survival end points \\
\hline CLEOPATRA (28) & $\begin{array}{l}\text { Docetaxel Trastuzumab } \\
\pm \text { Pertuzumab }\end{array}$ & $\begin{array}{l}\text { HER2+ } \\
\text { First line }\end{array}$ & $\begin{array}{c}678 \text { (58 from metastatic } \\
\text { sites) }\end{array}$ & $\begin{array}{l}\text { For each } 10 \% \text { increase in sTILs OS adjusted HR 0.89, 95\% Cl: 0.83-0.96, } \\
P=0.0014 \\
\text { No significant association between TILs PFS (adjusted HR 0.95, 95\% Cl: } \\
0.90-1.00, P=0.063 \text { ) } \\
\text { Median TILs } 10 \%\end{array}$ \\
\hline Kashiwagi et al. (45) & Eribulin & All & 52 (all primary lesions) & $\begin{array}{l}\text { No significant difference in DFS between high and low-TIL groups }(P=0.489) \\
\text { TNBC: high TILs }(>10 \%) \text { group had significantly longer DFS than the low-TIL } \\
\text { group }(P=0.033)\end{array}$ \\
\hline Schmid et al. (48) & Atezolizumab & TNBC & 115 & $\begin{array}{l}\text { TILs }^{\mathrm{a}} \leq 10 \% \text { : median OS } 6.6 \text { months } \\
\text { TILs > 10\%: median OS } 12.6 \text { months }\end{array}$ \\
\hline Total patients & & & 845 & \\
\hline
\end{tabular}

DFS, disease-free survival; OS, overall survival; sTIL, stromal TIL, TNBC, triple-negative breast cancer.

${ }^{a}$ TILs were evaluated in this study as immune infiltrate as a percentage of tumor area and not as in the current recommendations.

from early-stage disease to the metastatic setting. An additional intriguing finding from this study is that TIL levels vary depending on the ethnicity of the patients implying that host factors may also be important determinants of antitumor immunity. This raises an additional possibility that stratification according to ethnicity, or at least documentation of ethnicity in clinical trials is needed to be able to tease out any effect of ethnicity on response to immuno-therapeutics.

The use of TILs in predicting response to eribulin in metastatic breast cancer has recently been evaluated (45). TILs were assessed in primary lesions at the time of breast cancer diagnosis in 52 patients. For TNBC, patients in the high-TIL group had significantly longer disease-free survival than TNBC patients in the low-TIL group. While limited by small numbers, the results of this study indicate that TILs may have a role in predicting response to eribulin in advanced TNBC.

These findings are consistent with the prognostic value of TILs that has been reported in the metastatic setting in other tumor types. Lymphocytic infiltrates in metastatic lesions have been found to be a prognostic marker in metastatic melanoma (46) and colorectal cancer (47). TILs in metastatic breast cancer, while are overall present at lower levels than primary disease, still maintain prognostic value. The advanced setting will be more complex than primary disease with immune heterogeneity likely existing at the different metastatic sites (28).

\section{CLINICAL UTILITY}

Tumor-infiltrating lymphocytes have potential clinical utility in breast cancer in a number of different domains. Since TILs are read on simple H\&E stained slides, it is not surprising that there is a tremendous interest to investigate their importance for the clinical setting.

We see the clinical utility of TILs in the following ways:

1. Prediction of responders to checkpoint blockade monotherapy.

Schmid et al. (48) recently reported the results of a phase 1a study of atezolizumab monotherapy in 115 metastatic TNBC patients. Higher overall response rates and longer overall survival were observed in patients with higher TIL levels. In the group with TILs $\leq 10 \%$, median OS was 6.6 months, compared with 12.6 months for those with TILs $>10 \%$. Of note, TILs here were evaluated differently and not as in the current guidelines. TILs were defined as immune infiltrate as a percentage of the tumor area. While further refinement of immune markers is likely, TILs evaluated on H\&E slides could represent an easy first step for many laboratories to determine which patients may benefit from checkpoint blockade as a single agent. Data in this area will undoubtedly increase in the next few years.

2. Identification of primary HER2+ and TNBC patients with excellent prognoses.

Tumor-infiltrating lymphocytes as a robust prognostic factor may potentially lead to the identification of those patients with excellent prognosis and thus may guide adjuvant treatment deescalation efforts. For example, in HER2+ patients with high-TIL levels, it may be appropriate to consider using only a taxane and trastuzumab rather than maximal therapy with combination anthracycline, taxane, trastuzumab, and pertuzumab (10). In the pooled analysis of 991 primary TNBC patients, those patients with node negative disease and TILs $20 \%$ or more (which is around the average level) had excellent 5-year distant metastases-free survival (92\% CI: 87-97\%) (Figure 1). TILs may have potential utility in the design of clinical trials, by appropriately grouping patients into those who may benefit from standard treatments, and those who require novel therapeutic strategies (49).

\section{Refinement of the neoadjuvant pCR endpoint.}

The neoadjuvant model represents an attractive model for drug development (50). What remains to be clearly elucidated is how the change in PCR induced by new agents will correlate with improvements in disease-free survival in the adjuvant setting $(51,52)$. Given that TILS are prognostic in the residual disease setting, we hypothesize that incorporation of TILS into the $\mathrm{pCR} /$ residual disease endpoint may improve prediction of those who do well, and help us understand how a novel agent performs. Incorporation into existing models such as residual 
cancer burden (53) may improve these surrogate endpoints as not all patients who do not achieve pCR relapse and identification of these patients that most benefit from the new agent will aid adjuvant trial design and statistical calculations.

\section{CAN WE CREATE A TIL RESPONSE WERE NONE EXISTS?}

A lack of the presence of antitumor immunity bodes for a poor prognosis and poor response to therapy. Therefore, a key area of interest is how to modulate the tumor microenvironment in order to convert a "low-TIL" tumor to a "high-TIL" tumor. Therapeutics could stimulate antigenicity and allow the augmentation of the antitumor immune response with checkpoint inhibitors. Potential approaches include multimodal combinations with chemotherapy, targeted therapies, radiotherapy, personalized vaccines, and adoptive T cell therapies (49).

The combination of conventional chemotherapeutic agents and immunotherapies is currently being investigated. A phase III trial of nab-paclitaxel combined with the anti-PD-L1 inhibitor atezolizumab in advanced TNBC is underway (ClinicalTrials.gov. NCT02425891), as is a phase III study of pembrolizumab plus chemotherapy (ClinicalTrials.gov NCT NCT02819518).

Ras-MAPK pathway activation has been described as a mechanism of promoting immune evasion in TNBC (43). Genetic alterations in Ras-MAPK signaling have been significantly associated with lower TIL levels. MEK inhibition can upregulate major histocompatibility complex and PD-L1 expression in TNBC cells both in vivo and in vitro. Thus, this combination is a very attractive hypothesis to investigate in patients. The combination of the MEK inhibitor cobimetinib, taxane chemotherapy, and atezolizumab is currently being investigated in advanced TNBC patients (ClinicalTrials.gov NCT02322814).

The efficacy of trastuzumab in HER2-positive breast cancer has traditionally been thought to be due to reductions in signaling downstream of the HER2 receptor. However, it is now understood that antibody-dependent cell-mediated cytotoxicity as well as $\mathrm{T}$ cell-mediated cytotoxicity play a central role in its action

\section{REFERENCES}

1. Dunn GP, Bruce AT, Ikeda H, Old LJ, Schreiber RD. Cancer immunoediting: from immunosurveillance to tumor escape. Nat Immunol (2002) 3(11):991-8. doi:10.1038/ni1102-991

2. Mittal D, Gubin MM, Schreiber RD, Smyth MJ. New insights into cancer immunoediting and its three component phases - elimination, equilibrium and escape. Curr Opin Immunol (2014) 27:16-25. doi:10.1016/j.coi.2014.01.004

3. Ravelli A, Roviello G, Cretella D, Cavazzoni A, Biondi A, Cappelletti MR, et al. Tumor-infiltrating lymphocytes and breast cancer: beyond the prognostic and predictive utility. Tumour Biol (2017) 39(4). doi:10.1177/1010428317695023

4. Ingold Heppner B, Loibl S, Denkert C. Tumor-infiltrating lymphocytes: a promising biomarker in breast cancer. Breast Care (Basel) (2016) 11(2): 96-100. doi:10.1159/000444357

5. Dushyanthen S, Beavis PA, Savas P, Teo ZL, Zhou C, Mansour M, et al. Relevance of tumor-infiltrating lymphocytes in breast cancer. BMC Med (2015) 13:202. doi:10.1186/s12916-015-0431-3

6. Yamaguchi R, Tanaka M, Yano A, Tse GM, Yamaguchi M, Koura K, et al. Tumor-infiltrating lymphocytes are important pathologic predictors for neoadjuvant chemotherapy in patients with breast cancer. Hum Pathol (2012) 43:1688-94. doi:10.1016/j.humpath.2011.12.013
(54-59). Studies of immunotherapies combined with anti-HER2 treatments are currently recruiting and the results are eagerly awaited (ClinicalTrials.gov NCT02318901, NCT02605915, NCT02924883).

\section{CONCLUSION}

Tumor-infiltrating lymphocytes, as evaluated on H\&E slides, has been shown in numerous studies now to be a reliable and reproducible marker of pre-existing antitumor immunity in breast cancer. It is clear that higher levels of TILs are associated with improved prognosis in early and advanced stage TNBC and HER2-positive breast cancer, as well as a higher probability of achieving pCR in the neoadjuvant setting. Analysis of TILs in residual disease specimens after neoadjuvant therapy has also been shown to have prognostic value. The evaluation of TILs as a biomarker in breast cancer is likely to be extended from the research domain to the clinical setting in the future.

As the body of evidence of the clinical validity of TILs grows, so too does the appreciation of the importance of the immune system in the biology and outcome of breast cancers. There remain considerable challenges in optimizing host antitumor immunity, and combination of checkpoint inhibitors with traditional and novel therapies is an area of active investigation.

\section{AUTHOR CONTRIBUTIONS}

Preparation/drafting of manuscript: LW, PS, SJL, BV, RS, and SL; figures: LW, RS, and SL.

\section{ACKNOWLEDGMENTS}

SJL is supported by the University of Melbourne. SL is supported by the Cancer Council Victoria John Colebatch fellowship, the Breast Cancer Research Foundation (BCRF) NY, the National Breast Cancer Foundation (NBCF) of Australia, and the National Health and Medical Research Council (NHMRC) of Australia.

7. Loi S, Sirtaine N, Piette F, Salgado R, Viale G, Van Eenoo F, et al. Prognostic and predictive value of tumor-infiltrating lymphocytes in a phase III randomized adjuvant breast cancer trial in node-positive breast cancer comparing the addition of docetaxel to doxorubicin with doxorubicin-based chemotherapy: BIG 02-98. J Clin Oncol (2013) 31(7):860-7. doi:10.1200/JCO.2011.41.0902

8. Ali HR, Provenzano E, Dawson SJ, Blows FM, Liu B, Shah M, et al. Association between CD8+ T-cell infiltration and breast cancer survival in 12439 patients. Ann Oncol (2014) 25:1536-43. doi:10.1093/annonc/mdu191

9. Ingold Heppner B, Untch M, Denkert C, Pfitzner BM, Lederer B, Schmitt W, et al. Tumor-infiltrating lymphocytes: a predictive and prognostic biomarker in neoadjuvant-treated HER2-positive breast cancer. Clin Cancer Res (2016) 22(23):5747-54. doi:10.1158/1078-0432.CCR-15-2338

10. Savas P, Salgado R, Denkert C, Sotiriou C, Darcy PK, Smyth MJ, et al. Clinical relevance of host immunity in breast cancer: from TILs to the clinic. Nat Rev Clin Oncol (2016) 13(4):228-41. doi:10.1038/nrclinonc.2015.215

11. Azim HA Jr, Michiels S, Zagouri F, Delaloge S, Filipits M, Namer M, et al. Utility of prognostic genomic tests in breast cancer practice: the IMPAKT 2012 Working Group Consensus Statement. Ann Oncol (2013) 24(3):647-54. doi:10.1093/annonc/mds645

12. Harris LN, Ismaila N, McShane LM, Andre F, Collyar DE, GonzalezAngulo AM, et al. Use of biomarkers to guide decisions on adjuvant systemic 
therapy for women with early-stage invasive breast cancer: American Society of Clinical Oncology Clinical Practice Guideline. JClin Oncol (2016) 34(10):1134-50. doi:10.1200/JCO.2015.65.2289

13. Salgado R, Denkert C, Demaria S, Sirtaine N, Klauschen F, Pruneri G, et al. The evaluation of tumor-infiltrating lymphocytes (TILs) in breast cancer: recommendations by an International TILs Working Group 2014. Ann Oncol (2015) 26(2):259-71. doi:10.1093/annonc/mdu450

14. Leung SCY, Nielsen TO, Zabaglo L, Arun I, Badve SS, Bane AL, et al. Analytical validation of a standardized scoring protocol for Ki67: phase 3 of an international multicenter collaboration. NPJ Breast Cancer (2016) 2:16014. doi:10.1038/npjbcancer.2016.14

15. Loi S, Drubay D, Adams S, Francis PA, Joensuu H, Dieci MV, et al. Pooled individual patient data analysis of tumor infiltrating lymphocytes (TILs) in primary triple negative breast cancer (TNBC) treated with anthracycline-based chemotherapy. San Antonio Breast Cancer Symposium. (2015). Publication Number: S1-03.

16. Brown $\mathrm{M}$, Wittwer $\mathrm{C}$. Flow cytometry: principles and clinical applications in hematology. Clin Chem (2000) 46:1221-9.

17. Stack EC, Wang C, Roman KA, Hoyt CC. Multiplexed immunohistochemistry, imaging, and quantitation: a review, with an assessment of Tyramide signal amplification, multispectral imaging and multiplex analysis. Methods (2014) 70:46-58. doi:10.1016/j.ymeth.2014.08.016

18. Desmedt C, Haibe-Kains B, Wirapati P, Buyse M, Larsimont D, Bontempi G, et al. Biological processes associated with breast cancer clinical outcome depend on the molecular subtypes. Clin Cancer Res (2008) 14(16):5158-65. doi:10.1158/1078-0432.CCR-07-4756

19. Denkert C, von Minckwitz G, Brase JC, Sinn BV, Gade S, Kronenwett R, et al. Tumor-infiltrating lymphocytes and response to neoadjuvant chemotherapy with or without carboplatin in human epidermal growth factor receptor 2-positive and triple-negative primary breast cancers. J Clin Oncol (2015) 33(9):983-91. doi:10.1200/JCO.2014.58.1967

20. Denkert C, Wienert S, Poterie A, Loibl S, Budczies J, Badve S, et al. Standardized evaluation of tumor-infiltrating lymphocytes in breast cancer: results of the ring studies of the international immuno-oncology biomarker working group. Mod Pathol (2016) 29(10):1155-64. doi:10.1038/ modpathol.2016.109

21. Swisher SK, Wu Y, Castaneda CA, Lyons GR, Yang F, Tapia C, et al. Interobserver agreement between pathologists assessing tumor-infiltrating lymphocytes (TILs) in breast cancer using methodology proposed by the International TILs Working Group. Ann Surg Oncol (2016) 23(7):2242-8. doi:10.1245/s10434-016-5173-8

22. Nawaz S, Heindl A, Koelble K, Yuan Y. Beyond immune density: critical role of spatial heterogeneity in estrogen receptor-negative breast cancer. Mod Pathol (2015) 28(6):766-77. doi:10.1038/modpathol.2015.37

23. Sobottka B, Pestalozzi B, Fink D, Moch H, Varga Z. Similar lymphocytic infiltration pattern in primary breast cancer and their corresponding distant metastases. Oncoimmunology (2016) 5(6):e1153208. doi:10.1080/21624 02X.2016.1153208

24. Cimino-Mathews A, Ye X, Meeker A, Argani P, Emens LA. Metastatic triple-negative breast cancers at first relapse have fewer tumor-infiltrating lymphocytes than their matched primary breast tumors: a pilot study. Hum Pathol (2013) 44(10):2055-63. doi:10.1016/j.humpath.2013.03.010

25. Ogiya R, Niikura N, Kumaki N, Bianchini G, Kitano S, Iwamoto T, et al. Comparison of tumor-infiltrating lymphocytes between primary and metastatic tumors in breast cancer patients. Cancer Sci (2016) 107(12):1730-5. doi:10.1111/cas.13101

26. Mansfield AS, Aubry MC, Moser JC, Harrington SM, Dronca RS, Park SS, et al. Temporal and spatial discordance of programmed cell death-ligand 1 expression and lymphocyte tumor infiltration between paired primary lesions and brain metastases in lung cancer. Ann Oncol (2016) 27(10):1953-8. doi:10.1093/annonc/mdw289

27. Baine MK, Turcu G, Zito CR, Adeniran AJ, Camp RL, Chen L, et al. Characterization of tumor infiltrating lymphocytes in paired primary and metastatic renal cell carcinoma specimens. Oncotarget (2015) 6(28): 24990-5002. doi:10.18632/oncotarget.4572

28. Luen SJ, Salgado R, Fox S, Savas P, Eng-Wong J, Clark E, et al. Tumourinfiltrating lymphocytes in advanced HER2-positive breast cancer treated with pertuzumab or placebo in addition to trastuzumab and docetaxel: a retrospective analysis of the CLEOPATRA study. Lancet Oncol (2017) 18(1):52-62. doi:10.1016/S1470-2045(16)30631-3

29. Loi S, Michiels S, Salgado R, Sirtaine N, Jose V, Fumagalli D, et al. Tumor infiltrating lymphocytes are prognostic in triple negative breast cancer and predictive for trastuzumab benefit in early breast cancer: results from the FinHER trial. Ann Oncol (2014) 25(8):1544-50. doi:10.1093/annonc/mdul12

30. Kim SR, Gavin PG, Pogue-Geile KL, Song N, Finnigan M, Bandos H, et al. A surrogate gene expression signature of tumor infiltrating lymphocytes (TILs) predicts degree of benefit from trastuzumab added to standard adjuvant chemotherapy in NSABP (NRG) trial B-31 for HER2+ breast cancer. AACR. (2015). abstract number 2837.

31. Adams S, Gray RJ, Demaria S, Goldstein L, Perez EA, Shulman LN, et al. Prognostic value of tumor-infiltrating lymphocytes in triple-negative breast cancers from two phase III randomized adjuvant breast cancer trials: ECOG 2197 and ECOG 1199. JClin Oncol (2014) 32(27):2959-66. doi:10.1200/ JCO.2013.55.0491

32. Pruneri G, Gray KP, Vingiani A, Viale G, Curigliano G, Criscitiello C, et al. Tumor-infiltrating lymphocytes (TILs) are a powerful prognostic marker in patients with triple-negative breast cancer enrolled in the IBCSG phase III randomized clinical trial 22-00. Breast Cancer Res Treat (2016) 158(2):323-31. doi:10.1007/s10549-016-3863-3

33. Loi S, Drubay D, Adams S, Francis PA, Joensuu H, Dieci MV, et al. Pooled individual patient data analysis of stromal tumor infiltrating lymphocytes in primary triple negative breast cancer treated with anthracycline-based chemotherapy. San Antonio Breast Cancer Symposium, San Antonio (2015). p. S1-03.

34. Amin MB, Edge S, Greene F, Byrd DR, Brookland RK, Washington MK, et al. The Eighth Edition AJCC Cancer Staging Manual: continuing to build a bridge from a population-based to a more "personalized" approach to cancer staging. CA Cancer J Clin (2017) 67(2):93-9. doi:10.3322/caac.21388

35. Denkert C, Loibl S, Noske A, Roller M, Müller BM, Komor M, et al. Tumor-associated lymphocytes as an independent predictor of response to neoadjuvant chemotherapy in breast cancer. J Clin Oncol (2010) 28(1):105-13. doi:10.1200/JCO.2009.23.7370

36. Loi S, Michiels S, Salgado R, Sirtaine N, Jose V, Fumagalli D, et al. Tumor infiltrating lymphocytes (TILs) indicate trastuzumab benefit in early-stage HER2-positive breast cancer (HER2+ BC). Cancer Res (2013) 73:aS1-05. doi:10.1158/0008-5472.SABCS13-S1-05

37. Dieci M, Bisagni G, Cagossi K, Bottinin A, Sarti S, Placentini F, et al. Tumor infiltrating lymphocytes and correlation with outcome in the Cher-LOB study. Cancer Res (2015) 75:aD1e1. doi:10.1158/1538-7445.SABCS14-PD1-1

38. Salgado R, Denkert C, Campbell C, Savas P, Nuciforo P, Aura C, et al. Tumorinfiltrating lymphocytes and associations with pathological complete response and event-free survival in HER2-positive early-stage breast cancer treated with lapatinib and trastuzumab: a secondary analysis of the NeoALTTO trial. JAMA Oncol (2015) 1(4):448-54. doi:10.1001/jamaoncol.2015.0830

39. Issa-Nummer Y, Darb-Esfahani S, Loibl S, Kunz G, Nekljudova V, Schrader I, et al. Prospective validation of immunological infiltrate for prediction of response to neoadjuvant chemotherapy in HER2-negative breast cancer - a substudy of the neoadjuvant GeparQuinto trial. PLoS One (2013) 8(12):e79775. doi:10.1371/journal.pone.0079775

40. West NR, Milne K, Truong PT, Macpherson N, Nelson BH, Watson PH. Tumor-infiltrating lymphocytes predict response to anthracycline-based chemotherapy in estrogen receptor-negative breast cancer. Breast Cancer Res (2011) 13(6):R126. doi:10.1186/bcr3072

41. Miyashita M, Sasano H, Tamaki K, Hirakawa H, Takahashi Y, Nakagawa S. Prognostic significance of tumor-infiltrating CD8+ and FOXP3+ lymphocytes in residual tumors and alterations in these parameters after neoadjuvant chemotherapy in triple-negative breast cancer: a retrospective multicenter study. Breast Cancer Res (2015) 17:124. doi:10.1186/s13058-015-0632-x

42. Dieci MV, Criscitiello C, Goubar A, Viale G, Conte P, Guarneri V, et al. Prognostic value of tumor-infiltrating lymphocytes on residual disease after primary chemotherapy for triple-negative breast cancer: a retrospective multicenter study. Ann Oncol (2014) 25(3):611-8. doi:10.1093/annonc/mdt556

43. Loi S, Dushyanthen S, Beavis PA, Salgado R, Denkert C, Savas P, et al. RAS/ MAPK activation is associated with reduced tumor-infiltrating lymphocytes in triple-negative breast cancer: therapeutic cooperation between MEK and PD-1/PD-L1 immune checkpoint inhibitors. Clin Cancer Res (2016) 22(6):1499-509. doi:10.1158/1078-0432.CCR-15-1125 
44. Arnedos M, Filleron T, Dieci MV, Adam J, Robbins PB, Loi S, et al. Genomic and immune characterization of metastatic breast cancer (MBC): and ancillary studies of the SAFIR01 \& M overall survival CATO trials. Ann Oncol (2014) 25(Suppl 4):iv116(abstr3510). doi:10.1093/annonc/mdu329.1

45. Kashiwagi S, Asano Y, Goto W, Takada K, Takahashi K, Noda S, et al. Use of Tumor-infiltrating lymphocytes (TILs) to predict the treatment response to eribulin chemotherapy in breast cancer. PLoS One (2017) 12(2):e0170634. doi:10.1371/journal.pone.0170634

46. Bogunovic D, O’Neill DW, Belitskaya-Levy I, Vacic V, Yu YL, Adams S, et al. Immune profile and mitotic index of metastatic melanoma lesions enhance clinical staging in predicting patient survival. Proc Natl Acad Sci U S A (2009) 106(48):20429-34. doi:10.1073/pnas.0905139106

47. Kwak Y, Koh J, Kim DW, Kang SB, Kim WH, Lee HS. Immunoscore encompassing CD3+ and CD8+ T cell densities in distant metastasis is a robust prognostic marker for advanced colorectal cancer. Oncotarget (2016) 7(49):81778-90. doi:10.18632/oncotarget.13207

48. Schmid P, Cruz C, Braiteh FS, Eder JP, Tolaney S, Kuter I, et al. Atezolizumab in Metastatic Triple-Negative Breast Cancer: Long-Term Clinical Outcomes and Biomarker Analyses. American Association for Cancer Research (2017).

49. Luen SJ, Savas P, Fox SB, Salgado R, Loi S. Tumour-infiltrating lymphocytes and the emerging role of immunotherapy in breast cancer. Pathology (2017) 49(2):141-55. doi:10.1016/j.pathol.2016.10.010

50. Gianni L, Pienkowski T, Im YH, Tseng LM, Liu MC, Lluch A, et al. 5-year analysis of neoadjuvant pertuzumab and trastuzumab in patients with locally advanced, inflammatory, or early-stage HER2-positive breast cancer (NeoSphere): a multicentre, open-label, phase 2 randomised trial. Lancet Oncol (2016) 17(6):791-800. doi:10.1016/S1470-2045(16)00163-7

51. Baselga J, Bradbury I, Eidtmann H, Di Cosimo S, de Azambuja E, Aura C, et al. Lapatinib with trastuzumab for HER2-positive early breast cancer (NeoALTTO): a randomised, open-label, multicentre, phase 3 trial. Lancet (2012) 379(9816):633-40. doi:10.1016/S0140-6736(11)61847-3

52. Piccart-Gebhart M, Holmes E, Baselga J, de Azambuja E, Dueck AC, Viale G, et al. Adjuvant lapatinib and trastuzumab for early human epidermal growth factor receptor 2-positive breast cancer: results from the randomized phase III adjuvant lapatinib and/or trastuzumab treatment optimization trial. J Clin Oncol (2016) 34(10):1034-42. doi:10.1200/JCO.2015.62.1797
53. Symmans WF, Wei C, Gould R, Yu X, Zhang Y, Liu M, et al. Long-term prognostic risk after neoadjuvant chemotherapy associated with residual cancer burden and breast cancer subtype. J Clin Oncol (2017) 35(10):1049-60. doi:10.1200/JCO.2015.63.1010

54. Clynes RA, Towers TL, Presta LG, Ravetch JV. Inhibitory Fc receptors modulate in vivo cytotoxicity against tumor targets. Nat Med (2000) 6(4):443-6. doi: $10.1038 / 74704$

55. Kroemer G, Senovilla L, Galluzzi L, Andre F, Zitvogel L. Natural and therapy induced immunosurveillance in breast cancer. Nat Med (2015) 21(10):1128e38. doi:10.1038/nm.3944

56. Musolino A, Naldi N, Bortesi B, Pezzuolo D, Capelletti M, Missale G, et al. Immunoglobulin $\mathrm{G}$ fragment $\mathrm{C}$ receptor polymorphisms and clinical efficacy of trastuzumab-based therapy in patients with HER-2/neu-positive metastatic breast cancer. J Clin Oncol (2008) 26(11):1789e96. doi:10.1200/JCO.2007.14.8957

57. Tamura K, Shimizu C, Hojo T, Akashi-Tanaka S, Kinoshita T, Yonemori K, et al. FcgammaR2A and 3A polymorphisms predict clinical outcome of trastuzumab in both neoadjuvant and metastatic settings in patients with HER2-positive breast cancer. Ann Oncol (2011) 22(6):1302e7. doi:10.1093/annonc/mdq585

58. Norton N, Olson RM, Pegram M, Tenner K, Ballman KV, Clynes R, et al. Association studies of Fcgamma receptor polymorphisms with outcome in HER2+ breast cancer patients treated with trastuzumab in NCCTG (Alliance) Trial N9831. Cancer Immunol Res (2014) 2(10):962e9. doi:10.1158/2326-6066.CIR-14-0059

59. Park S, Jiang Z, Mortenson ED, Deng L, Radkevich-Brown O, Yang X, et al. The therapeutic effect of anti-HER2/neu antibody depends on both innate and adaptive immunity. Cancer Cell (2010) 18(2):160e70. doi:10.1016/j.ccr.2010.06.014

Conflict of Interest Statement: The authors declare that the research was conducted in the absence of any commercial or financial relationships that could be construed as a potential conflict of interest.

Copyright (c) 2017 Wein, Savas, Luen, Virassamy, Salgado and Loi. This is an openaccess article distributed under the terms of the Creative Commons Attribution License (CC BY). The use, distribution or reproduction in other forums is permitted, provided the original author(s) or licensor are credited and that the original publication in this journal is cited, in accordance with accepted academic practice. No use, distribution or reproduction is permitted which does not comply with these terms. 